\title{
Relationship between cognitive decline and depression in patients with Parkinson's disease
}

\author{
Denisa Floriana Vasilica Pirscoveanu', Valerica Tudorica', Cornelia Zaharia², \\ Carmen Valeria Albu', Diana Iulia Stanca', Puiu Olivian Stovicek ${ }^{3}$, Mircea Pirscoveanu ${ }^{1}$ \\ ${ }^{1}$ University of Medicine and Pharmacy, Craiova, Romania \\ ${ }^{2}$ Clinical Hospital of Neuropsychiatry, Craiova, Romania \\ ${ }^{3}$ Titu Maiorescu University, Tirgu Jiu, Romania
}

\begin{abstract}
The aim of this study was to assess the cognitive decline in patients with Parkinson's disease and to study its correlation with their depression.

We studied 64 consecutive patients (30 men and 34 women), mean education level $11.2 \pm 1$ years and mean age $72.3 \pm 3.5$ years. These patients were admitted to Clinic of Neuropsychiatry Craiova between January 2016 and October 2016.

They underwent a neurological and neuropsychological examination at baseline, after 6 months and 12 months later. For the cognitive assessment we used the revised version of the Addenbrooke's Cognitive Examination (ACE-R) and Montreal Cognitive Assessment (MoCA) and for the evaluation of depression we used Hamilton Depression Rating Scale (HDRS).

We have also included in our study a control group composed of 76 control subjects with the same range of educational level and age. All the patients met the diagnostic criteria for Parkinson's Disease. In this study we included patients in stage I and II on Hoehn and Yahr scale. Twenty-six patients were in stage I and 38 patients in stage II. The patients were treated with levo-dopa monotherapy, dopaminergic agonists monotherapy, or levodopa associated with dopaminergic agonists. At baseline the patients in stage I showed a mean MoCA score 26.8 and a mean ACE-R score 89.6. The patients in stage II had a mean MoCA score 26.5 and a mean ACE-R score 89.4 at baseline.

The control subjects showed a mean MoCA score 27.06 and a mean ACE-R score 90.3. One year later the patients group I showed a mean MoCA score 19.8 and 84.7 mean ACE-R score. The groups of patients in stage II had a mean MoCA score 18.3 and ACE-R score 84.2. The control group the mean MoCA values was 22.56 and ACE-R 87.1. Regarding asssessment of depression, we obtained the next scores: at baseline, patients in stage I showed a mean HDRS score of 14.6; the patients in stage II a mean HDRS score 14.8 and the control group a HDRS mean score of 8. One year later group I showed a mean HDRS score of 18.8; the group II 19.9 and the control group 9.7.

For the statistical analyse, we used Student test $(p<0.05$ statistically considerable).

The patients with PD showed a greater cognitive impairment and depression than the control subjects. We observed that the patients in stage II Hoehn and Yahr had a greater cognitive decline and depression than patients in stage I. Depression was diagnosed in 40 patients at 6 months and in 56 of the patients at 12 months. The cognitive impairment was higher at 12 months than at 6 months. The depressive patients had more severe cognitive decline than the nondepressive patients.
\end{abstract}

Keywords: cognitive decline, Parkinson's disease, depression

\section{INTRODUCTION}

Parkinson's disease (PD) is a neurodegenerative disorders which affects about $1 \%$ of the population over age 60 (1).

PD is characterized by tremor, rigidity, bradykinesia and impairment of balance that are usually of an asymmetric course (2).
In addition to motor deficits, non-motor symptoms in PD received increasing attention (3). Cognitive decline is included in non-motor features of PD (3). Also the mood disorders, psychosis, sleep disturbances, and autonomic dysfunction are included features of PD (4). These non-motor features have been associated with increased disability and reduced quality of life (5). 
In Parkinson's disease we encounter a variety of symptoms of cognitive impairment starting from stage of mild cognitive impairment (MCI) to dementia (6). Diagnostic criteria for MCI include subjective complaining from the patient regarding cognitive decline, minimal effect of the decline on day to day functioning and the absence of dementia, and evidence of cognitive decline that cannot be attributed to age $(7,8)$. The exact cause of cognition impairment in PD are not fully known but are likely due to a combination of chemical and structural changes.

To diagnose MCI in PD patients, cognitive impairment should occur within the context of clinically diagnosed PD (9).

Depression is the most frequent psychiatric disorder in PD patients (10). Approximately 50\% of these patients are suffering of depression (11). The depression might not be detected when occuring with PD, thus, the number of cases might even be higher (12).

The depression may lead to pseudodementia which may be difficult to distinguish from the primary cognitive disturbacens in PD (13).

Diagnosis of depression in PD may be difficult because of overlaping symptoms of the two disorders (14). Symptoms as depression and anxiety may occur before manifestation of motor symptoms (15). The diagnosis of depression is based on subjectively experienced symptoms including feelings of emptiness, reduced emotional reactivity and inability to experience pleasure (16).

Both depression and cognitive impairment in $\mathrm{PD}$, have major clinical consequences including a negative impact of quality of life.

It seems that motor symptoms of the disease manifest earlier in patients with PD and depression (17). The patients with depression and PD do not regards depression as a disease, but they consider that their depressed mood is a regular feature of PD (18).

Most studies do not show a relationship between gender of PD patients and frequency of depression (19).

The non-motor symptoms become even more important as Parkinson's disease advances, so the recognition and treatments for these symptoms are needed.

\section{OBJECTIVE}

Our study was designed to assess the cognitive decline in Parkinson's disease and to study its correlation with depression.

\section{MATERIAL AND METHODS}

We included in these study 64 patients with Parkinson's disease (group I composed of 26 patients in stage I Hoehn and Yahr, group II composed of 38 patients in stage II), and a group control consisted of 76 subjects with the same range of educational level and age.

In the group patients were 30 men and 34 women, with a mean education level $11.2 \pm 1$ years and mean age $72.3 \pm 3.5$ years. These patients were admitted to Clinic of Neuropsychiatry Craiova between January 2016 and October 2016. All the patients met the diagnostic criteria for Parkinson's disease. In control group the mean age was $71.9 \pm 3$ years and a mean education level $11.5 \pm 6$ years.

The patients were treated with levo-dopa monotherapy (24 patients), dopaminergic agonists monotherapy (20 patients), or levodopa associated with dopaminergic agonists (20 patients).

Upon giving an informed consent, regarding cognitive assessment, patients and control groups were tested using MoCA and ACE-R. For the assessment of depression we used HDRS scale both in patients and control groups. Our evaluations were made at the beginning of the study (at baseline), then after 6 months, respectively 12 months.

We compared the cognitive scores obtained in the patients group with those from control group and we have also followed-up which are the most impaired cognitive functions. The cognitive performances and the depressive state were related to the antiparkinsonian therapy. Then, we correlated the cognitive scores with the scores obtained in dynamics at the assessment of depression, both in patients and control groups.

The results were analysed by Student Test $(p<0.05$ statistically considerable).

\section{RESULTS}

Depression was diagnosed in 40 patients at 6 months and in 56 of the patients at 12 months. The patients included in our study showed a moderate depression, both in stage I and stage II. 
The depression assessment using HDRS showed in control group a mean score of 6.5 at baseline, 6.8 after 6 months and 7.1 at the end of study. At the beginning of the study, the PD patients in stage I Hoehn and Yahr had mean HDRS score of 11.6; after 6 months 13.8 and at endline 15.8.

The group of PD patients with stage II of the disease had at baseline a HDRS score of 11.9; at 6 months 14.8 and one year later 17 (Fig. 1).

The mean MoCA values obtained in dynamics in PD patients stage I were: 26.8 at baseline, 21.5 at
6 months and 19.8 one year later. In patients with stage II of the disease, mean MoCA scores were at baseline 26.5, at 6 months 20.5 and after one year 18.3. The control group showed at baseline a mean MoCA score of 27.06; at 6 months 24.5 and 22.56 at the end of study (Fig. 2).

Assessing the cognitive state using ACE-R, the group of patients with stage I showed a mean ACE-R score at baseline of 89.6; after 6 months 87.1 and mean value of 84.7 at the end of the study. In the group of study including patients with stage

\section{HDRS score}

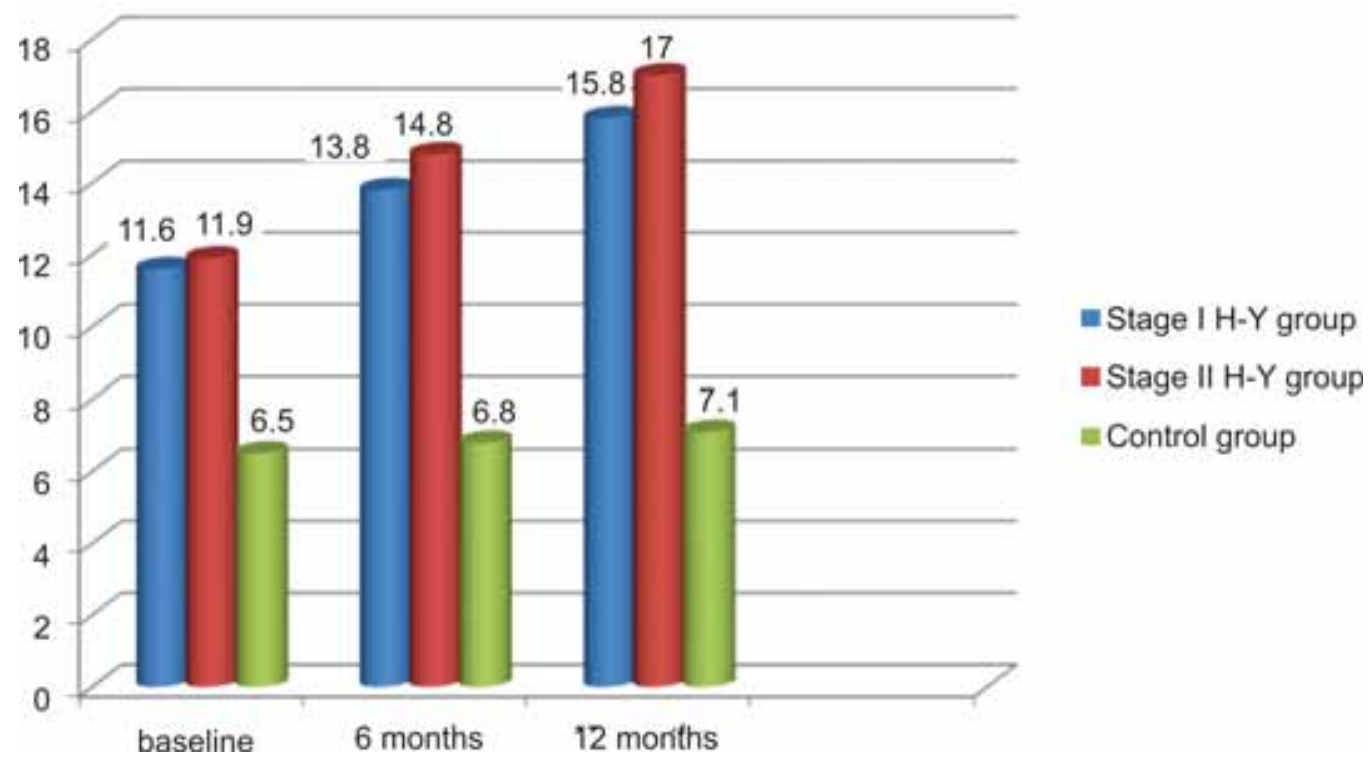

FIGURE 1. HDRS score in dynamics in patients and control groups $(p>0.05)$

\section{MoCA score}

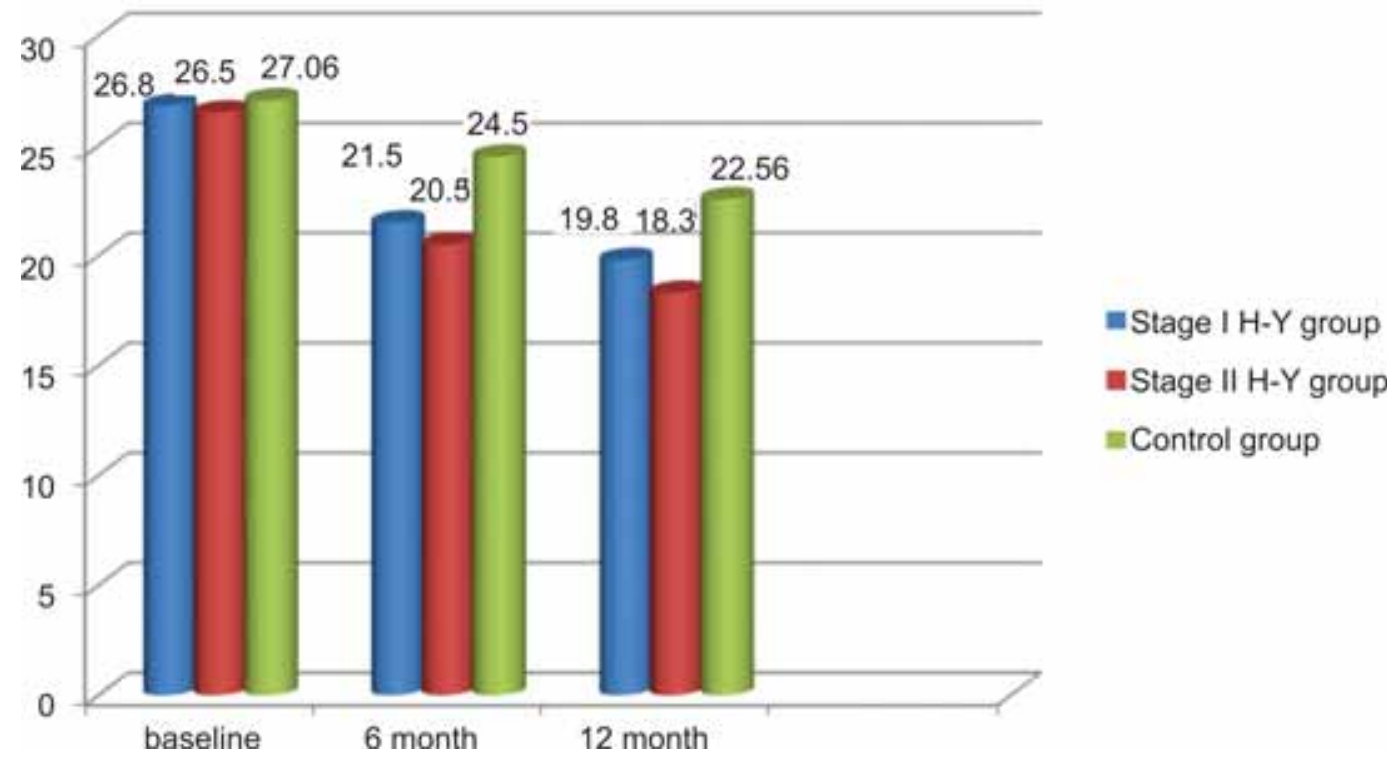

FIGURE 2. Cognitive assessment using MoCA in patients and control groups $(p<0.05)$ 
II, the mean values of ACE-R score were: 89.4 at baseline; 86.36 months later and 84.2 at endline. The control group showed at the beginning of the study a mean ACE-R score of 90.3; at 6 months 89.2 and 87.1 after one year (Fig. 3).

In patients of our study, we found that most affected cognitive domains were: executive function, visuospatial abilities, language, praxis, attention and verbal fluency (Fig. 4).

$61.4 \%$ of the patients trated with levodopa monotherapy showed a cognitive impairment while
$54.2 \%$ of the patients treated with dopamine agonists and $56.4 \%$ of the patients treated with dopamine agonists and levodopa associated. In Fig. 5 and no 6 we showed the evolution of MoCA and ACE-R scores related to antiparkinsonian treatment.

Regarding depressive state related to antiparkinsonian therapy, we observed that patients treated with levodopa were more depressive in comparison to patients treated with dopamine agonists or associated therapy (Fig. 7).

ACER score

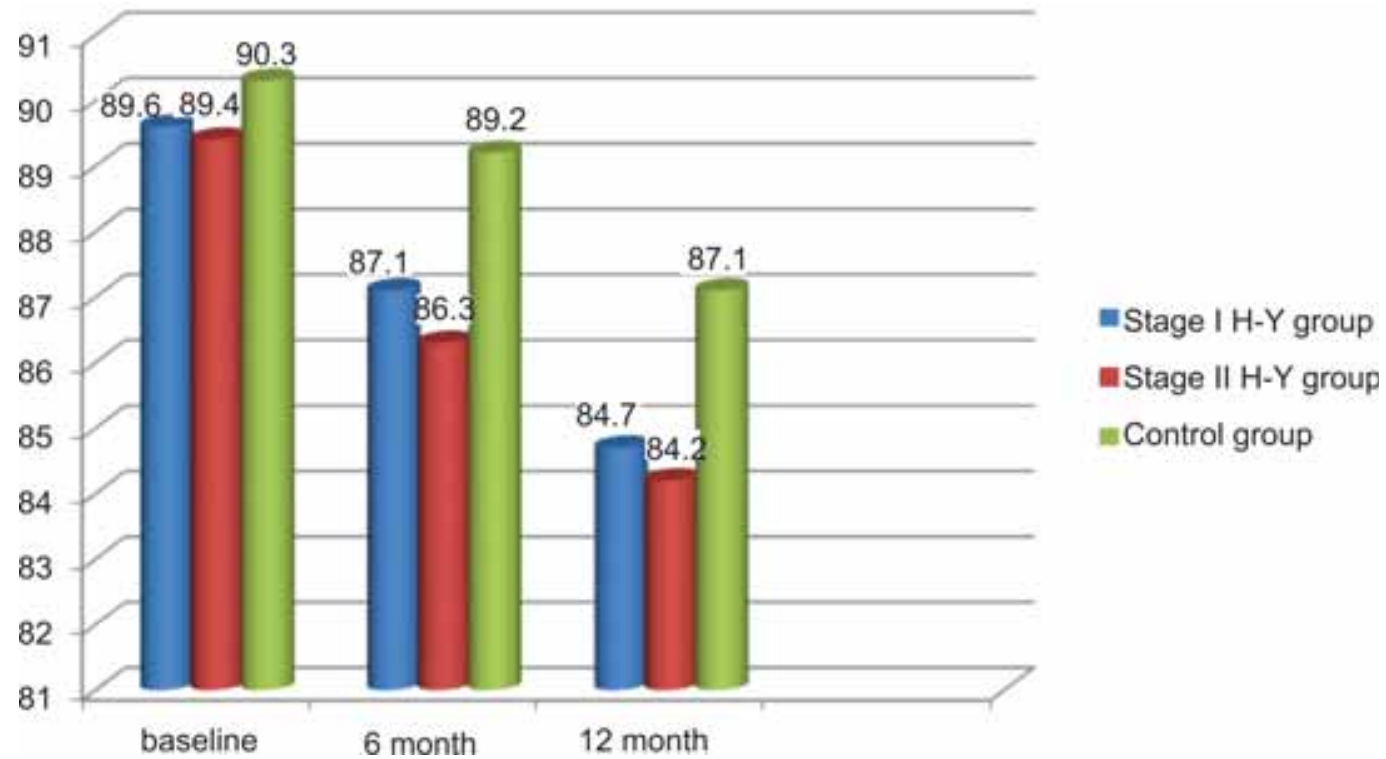

FIGURE 3. Cognitive assessment using ACER in patients and control groups $(p<0.05)$

\section{Cognitive domains affected}

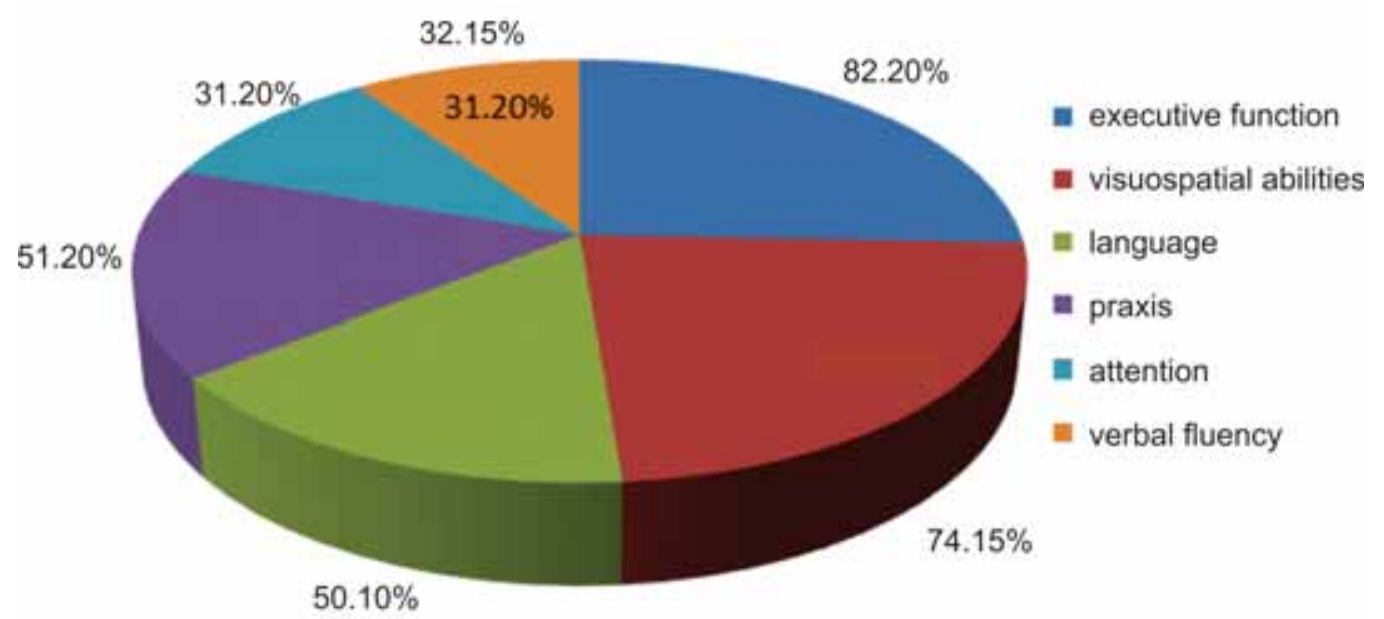

FIGURE 4. Most cognitive domains affected in PD patients 


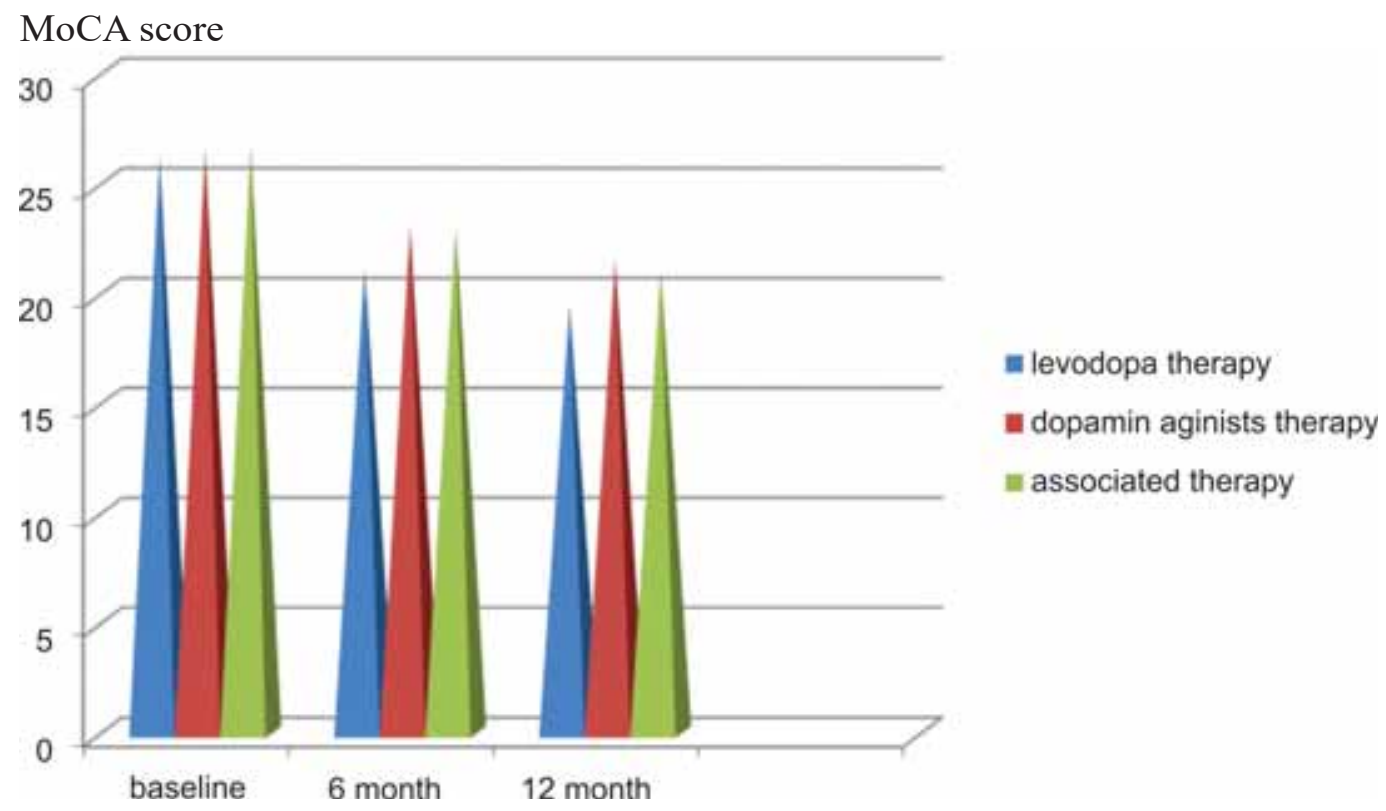

FIGURE 5. Mean MoCA scores related to the type of antiparkinsonian therapy $(p>0.05)$

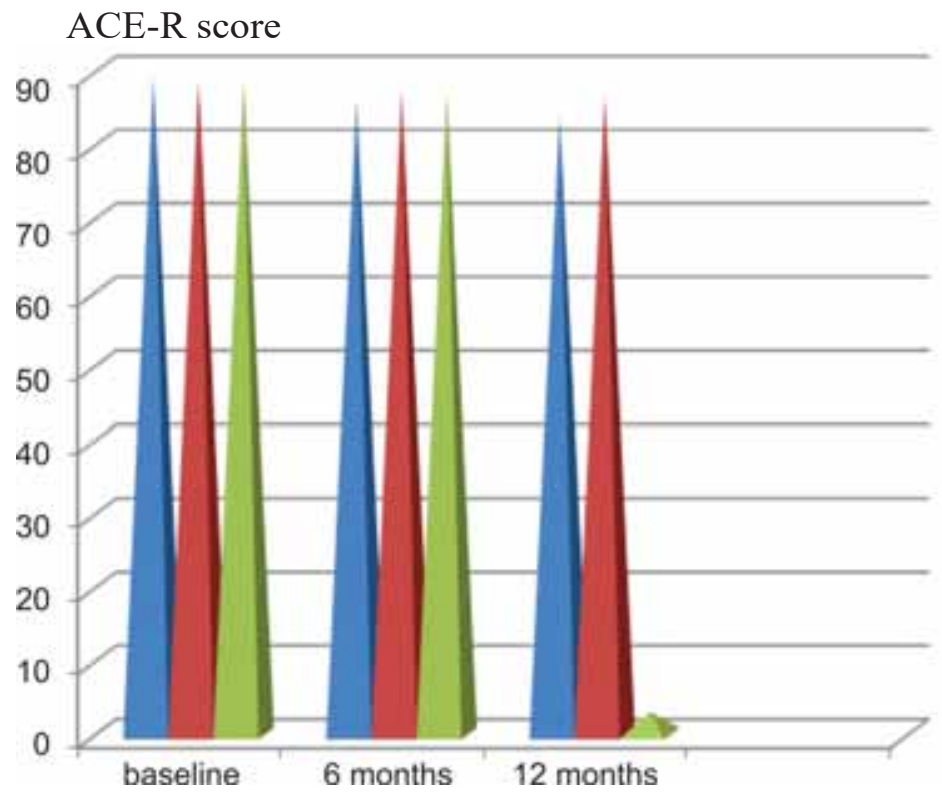

* levodopa therapy

E dopamine agonist theraly

m associated therapy

FIGURE 6. Mean ACE-R scores related to the type of antiparkinsonian therapy $(p>0.05)$

HDRS score

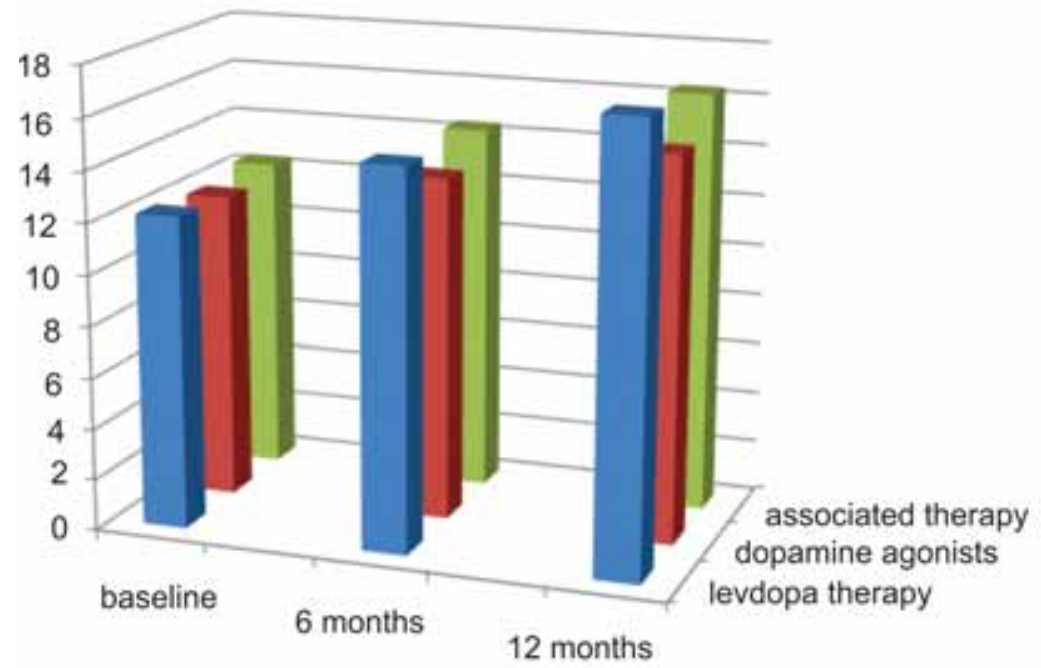

= levodopa therapy

Edopamine agonists

= associated therapy

FIGURE 7. Depression assessment related to the type of antiparkinsonian therapy ( $p>0.05)$ 


\section{DISCUSSIONS}

Although both depression and cognitive decline are frequent in PD patients, little is known on how the association of depression and PD varies according to cognitive decline. It seems that depression in PD occurs independently of dementia.

Studying our 64 patients wih PD in stage I and II Hoehn and Yahr, we observed an increase of depressive state during one year study.

As the figure 1 shows, the stage II patients were more depressives than patients in stage I and controls, but statistically insignificant $(\mathrm{p}>0.05)$. Both stage I and II patients started in the study having a mild depression. In the dynamics, their depressive state has evolved differently depending on the stage of PD. So, at six months evaluation we noticed that patients in stage I were still in mild depression, while patients in stage II had moderate depression. After a year of study, patients in both stages had moderate depression, but we observed that those in the stage II had a higher HDRS score than those in stage I.

We mention that a limitation of our study is that diagnosis of depression may have been influenced by the severity of bradykinesia in PD patients.

A weak point of this study is the fact that a small number of patients with PD are included. That is why we intend to carry out much more elaborate studies in the future, including a larger number of patients, to achieve more representative results.

Another weak point we recognize in this study is the relatively short period it has been unfolding (one year). So, our aim is to carry out a similar study in the future that will last for a long time and in which patients are evaluated with scales that include a much wider range of cognitive domains

In our study we observed a decrease of the cognitive performances in dynamics during the year study both in group of PD patients and control group. Using MoCA and ACE-R for the cognitive assessment, we observed that the $\mathrm{PD}$ patients showed a higher cognitive impairment than the control group, after both 6 months and one year $(p<0.05)$. In the figures number 2 and number 3 we described the graphics of these findings. Our results are in concordance with other studies that described a cognitive impairment in patients with $\mathrm{PD}$, and that declare that the risk for the development of de- mentia in PD patients is approximately 6 times higher than compared to non PD matched controls. $(7,20)$ Is well known that mild cognitive impairment in PD is a distinct entity and a potential prodromal state to PD dementia.

Generally it is assumed that cognitive impairment may develop early in the disease, but clinical symptoms of dementia appear only on late course of the disease (21).

At the start of our study, both stage I and stage II had a cognitive similarity in MoCA and ACE-R scores. In patients with stage II of the disease, the cognitive decline was significantly increased after 6 months and 12 months respectively, compared to the patients in stage I $(p<0.05)$.

The relationship between Hoehn and Yahr stage and cognitive impairment has long been described in PD. Our findings are in concordances with other studies that found the same conclusion $(22,2)$. We observed that cognitive decline was greater in patients with moderate depression than in patients with mild depression or no depression.

Cognitive decline in non demented PD patients encompasses a broad spectrum of clinical deficits and severity. It is known a difficulty with multi-tasking or planning, decreased attention and word finding disturbances (23). Regarding cognitive domains affected in PD patients, we found that executive function was most affected $(82.20 \%$ of the patients group). The second place in terms of cognitive impairment was visuospatial abilities followed by language disorders, praxis, attention and verbal fluency. Their percentage values are described in figure number 4 . Our findings are in concordance with results of other studies that found executive function as the most frequent cognitive domain affected in nondemented PD patients (23). Memory functions seem to be relatively preserved in patients with PD and cognitive decline.

When we studied the relationship between the therapy of Parkinson's disease and the range of the cognitve decline we did observed that both MoCA and ACE-R scores are related to antiparkinsonian treatment. So we discovered during year of study that both scores MoCA and ACE-R are more increased in patients levodopa treated in comparison to patients treated with dopamine agonists or associated therapy (Fig. 5, 6). The group patients treated with dopamine agonists and the group treated 
with associated therapy showed similar cognitive performances.

So both groups of patients experienced cognitive decline but lower intensity compared to levodopa treated patients.

Regarding correlation of depressive status with antiparkinsonian therapy received by PD patients, we observed that levodopa-treated patients experienced a more depressive state at 6 and 12 months than those treated with dopamine agonists or associated therapy (Fig. 7).

\section{CONCLUSIONS}

Our study showed that PD patients presented in dynamics a cognitive impairment. Also we found that the depression status of patients is increasing as the disease progresses. We observed a faster cognitive decline among depressed patients than nondepressed patients with PD.

It is hoped that more studies may clarify the neuropathological changes underlying the association of depression and cognitive decline in PD.

Conflict of interest: none declared Financial support: none declared

\section{REFERENCES}

1. Chandhiri K.R, Healy D.G, Schapira A.H. Non motor symptoms of Parkinson's disease: diagnosis and management. Lancet. Neurol 2006; 5: 235-245

2. Aarsland D., Zaccai J., Brayne C. A systematic review of prevalence studies of dementia in Parkinson's disease. Mov Disord. 2005; 20(10):1255-1263.

3. Stennis G.W., Leverenz J.B. Profile of cognitive impairment in Parkinson's disease. Brain Pathol 2010; 20(3) : 640-645

4. Galvin J.E. Cognitive change in Parkinson's disease. Alzheimer Dis Assoc Disord. 2006; 20(4):302-310.

5. Aarsland D., Bronnick K., Larsen J.P., Tysnes O.B., Alves G. Cognitive impairment in incident, untreated Parkinson's disease: the Norwegian ParkWest study. Neurology. 2009; 72(13):1121-1126

6. Emre M. Dementia associated with Parkinson's Disease. Lancet Neurol 2003; 2: 229-237.

7. Petersen R.C. Mild cognitive impairmentas a diagnostic entity. J Intern Med. 2004; 256:183-194

8. Petersen R.C., Roberts R.O., Knopman D.S., Boeve B.F., Geda Y.E., Ivnik R.J., Smith G.E., Jack C.R. Jr. Mild cognitive impairment: ten years later. Arch Neurol 2009; 66:1447- 1455

9. Pirscoveanu D., Tudorica V., Zaharia C., Matcau D., Stanca D., Trifan F. One year follow up studyon cognitive performances in patients with Parkinson's disease. Romanian Journal of Neurology. 2009; 8(2): 84-88

10. Cummings J.L., Masterman D.L. Depression in patients with Parkinson's disease. Int J Geriatr Psychiatry. 1999; 14: 11-718

11. Starkestein S.E., Petracca G., Chemerinski E., Merello M. Prevalence and correlates of parkinsonism in patients with primary depression. Neurology 2001; 57: 553-555

12. Lemke M.R. Depression in Parkinson's disease: Clinical features and treatment. Focus on Parkinson's disease. 2003; 5:3-8

13. Cummings J.L. Depression and Parkinson's disease: a review. Am J Psychiatry 1992; 149: 443-454

14. Lemke M.R. Motor phenomena in depression. Nervenarzt 1999; 70: $600-612$

15. Lemke M.R. Neuropsychiatric aspects of Parkinson's disease. Focus on Parkinson's disease 2004; 6: A44-A48

16. Starkestein S.E., Preziosi T.J., Bolduc P.L., Robinson R.G. Depression in Parkinson's disease. J Nerv Ment Dis 1990; 178: 27-31

17. Yamamoto M. Dpepression in Parkinson's disease: its prevalence, diagnosis, and neurochemical background. J Neurol 2001; 248 Suppl 3: III5-11

18. Allain H., Schuck S., Mauduit N. Depression in Parkinson's disease. BMJ 2000; 320: 1287-1288

19. Richard I.H. Depression in Parkinson's disease. Curr Treat Options Neurol 2000; 2: 263-274

20. Aarsland D., Andersen K., Larsen J.P. et al. Prevalence and characteristics of dementia in Parkinson's disease: an 8 year prospective study. Arch Neurol 2003; 60:387-92

21. Fuchs G.A., Gemende I., Herting B. et al. Dementia in idiopathic Parkinson syndrome. J Neurol 2004; 251(Suppl 6)VI/ 28-32.

22. Siciliano M., Mico R., Trojano L., De Stefano M., Baiano C., Passaniti C., De Mase A., Russo A., Tedeschi G., Tessitore A. Cognitive impairment is associated with Hoehn and Yahr stages in early de novo Parkinson's disease patients. Parkinsonism and Related Disorders 2017; 41:86-91

23. Goldman J.G., Litvan I. Mild cognitive impairment in Parkinson's disease, Minerva Med 2011; 102(6): 441-459 